\title{
Prominence-like Clouds Near HK Aqr
}

\author{
G.H.J. van den Oord
}

Sterrekundig Instituut, Postbus 80.000, 3508 TA Utrecht, The Netherlands

\author{
P.B. Byrne and M.T. Eibe \\ Armagh Observatory, College Hill, Armagh BT61 9DG, N. Ireland
}

\begin{abstract}
A time series of $\mathrm{H}_{\alpha}$ spectra of the rapidly rotating star $\mathrm{HK}$ Aqr has been analyzed. Evidence is found for the presence of cool clouds which are in co-rotation with the star. The cloud velocities, as derived from the clouds' absorption features, can be used to put constraints on the clouds' co-latitudes and their distances from the star using a socalled visibility diagram. For HK Aqr most clouds are at distances of 2-3 stellar radii and do not extend beyond the co-rotation radius. By using a simple radiative transfer model, we demonstrate that for most stars the presence of clouds affects the whole $\mathrm{H}_{\alpha}$ profile and does not result in discrete absorptions. Only clouds near rapidly rotating stars, with an inclination close to $90^{\circ}$, will cause discrete absorption features. The cool cloud plasma can form when a temperature inversion is created at the apex of a stellar-sized coronal loop because of reduced coronal heating at large distances from the star. It is likely that the cloud condensations are related to inverse polarity flaments because, near rapidly rotating stars, the axial current in normal polarity filaments decreases with height and has to change sign at the co-rotation radius.
\end{abstract}

\section{Introduction}

Observations of the $\mathrm{H}_{\alpha}$ profile of the rapidly rotating M1.5 dwarf star HK Aqr $\left(P_{\text {rot }}=10.34 \mathrm{~h}\right)$ indicate the presence of cool clouds which are forced into corotation by the stellar magnetic field. These clouds cause absorption features which transit the underlying $\mathrm{H}_{\alpha}$ profile from blue to red. Observations of $\mathrm{HK}$ Aqr have previously been reported by Byrne et al. (1996). In van den Oord et al. (1997, VDOBE) we present a further analysis of the cloud phenomenon.

\section{Detection of the Clouds}

When a point-like cloud is in front of the stellar disk it causes an absorption at velocity

$$
v_{a b s}=\Omega R_{c} \sin \theta_{c} \sin i \sin \varphi
$$

with $\Omega=2 \pi / P_{\text {rot }}$ the angular velocity of the star, $R_{c}$ the distance of the cloud to the center of mass of the star, $\theta_{c}$ the co-latitude of the cloud, $i$ the inclination 


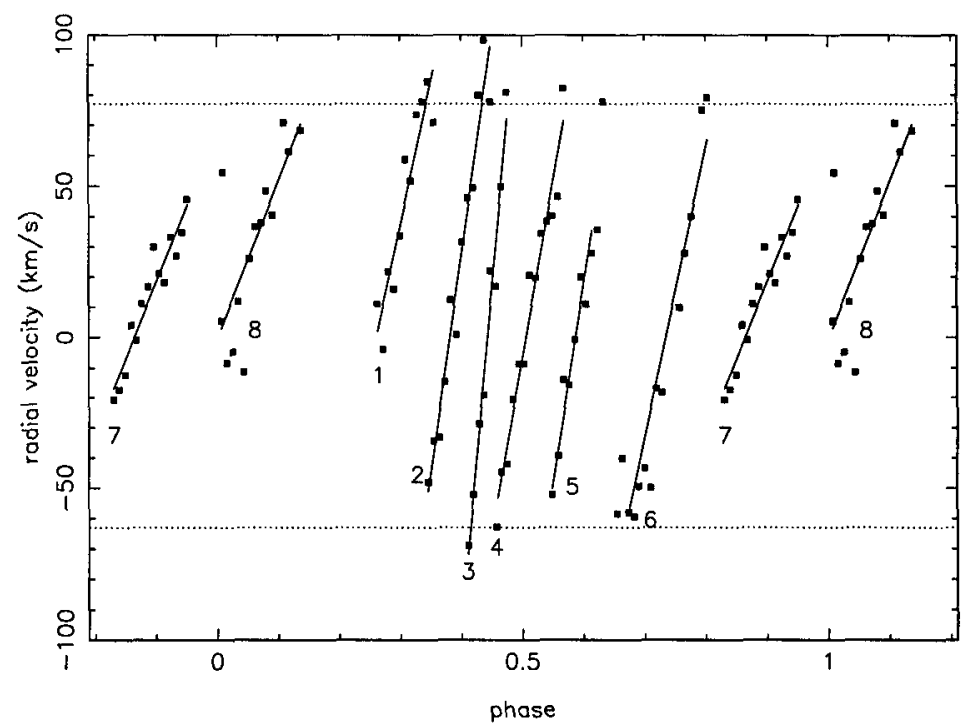

Figure 1. Observed radial velocity tracks for HK Aqr's clouds as a function of orbital phase. The observing conditions for clouds 7 and 8 were affected by the occurrence of a flare. Cloud 1 was already crossing the disk when the observations started.

of the star and $\varphi=\Omega t$ the phase angle of the cloud. At central meridian crossing $(\sin \varphi=0) \mathrm{Eq}$. (1) gives

$$
\frac{R_{\mathrm{c}} \sin \theta_{\mathrm{c}}}{R_{*}}=\left.\frac{1}{\Omega} \frac{1}{v_{*} \sin i} \frac{d v_{a b s}}{d t}\right|_{\sin \varphi=0}
$$

where $v_{*}=\Omega R_{*}$ is the intrinsic equatorial rotation speed of the star. The right hand side of Eq. (2) can be determined from observations, thus providing information about $R_{\mathrm{c}} \sin \theta_{c} / R_{*}$. The phases of in- and egress of a cloud ( $\varphi \equiv$ $\pm \Psi)$ are given by

$$
\cos \Psi=\left(\sqrt{1-R_{*}^{2} / R_{c}^{2}}-\cos \theta_{c} \cos i\right) /\left(\sin \theta_{c} \sin i\right) .
$$

Because Eqs. (2) and (3) depend on $R_{c} / R_{*}$ and on the cloud's co-latitude $\theta_{c}$, it is possible to construct a so-called visibility diagram depicting contours of $R_{c} \sin \theta_{c} / R_{*}$ and $\cos \Psi$. In Figure 1 we show the observed radial velocity tracks of eight clouds which have been identified in the $\mathrm{H}_{\alpha}$ spectra of HK Aqr. In Figure 2 we show the positions of the clouds in the visibility diagram. Most clouds are located below the co-rotation radius.

Subtracting the observed spectra from a template spectrum gives the absorbed flux. This flux can be written as

$$
F_{a b s}(\Delta \lambda)=\int_{-1}^{1} \mathcal{K}(\Delta \lambda-\Lambda y) Z(y) d y
$$




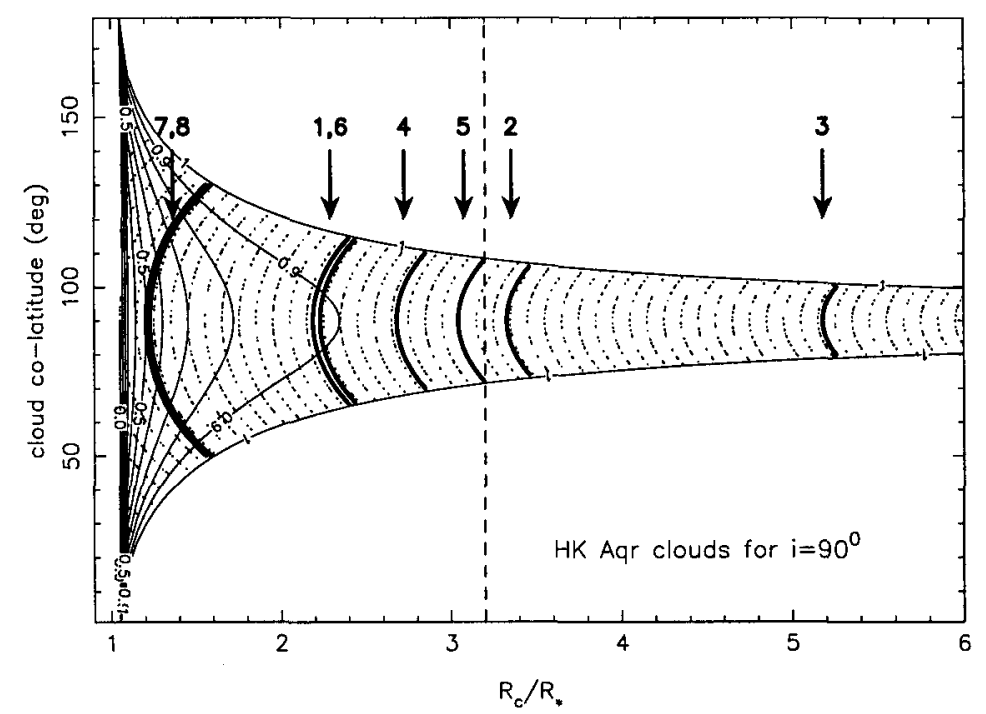

Figure 2. Visibility diagram for the clouds observed on HK Aqr. The thick solid lines indicate the possible locations of the clouds. The labels correspond to the numbers in Figure 1. The dashed line indicates the location of the co-rotation radius. The thin solid contours indicate $\cos \Psi=$ constant and the dotted contours $R_{c} \sin \theta_{c} / R_{*}=$ constant. Clouds in the blank area are off the disk and do not result in absorptions.

where $\Delta \lambda=\lambda-\lambda_{c}, \lambda_{c}$ is the central wavelength of $\mathrm{H}_{\alpha}, \Lambda=\left|v_{*} \sin i\right| \lambda_{c} / c$, $\mathcal{K}(\Delta \lambda)=I_{*}(\Delta \lambda)[1-\exp \{-\tau(\Delta \lambda)\}], I_{*}$ is the stellar line-of-sight intensity and $Z(y)$ is a function describing the distribution of the clouds on the projected stellar disk. Lines $y=$ constant correspond to a specific line-of-sight velocity. The total cloud area is given by $\int Z(y) d y$. Eq. (4) shows that the absorbed flux is given by a convolution of $Z(y)$ with the kernel $\mathcal{K}$. The accuracy with which $Z(y)$ can be recovered depends on the width of the kernel $\mathcal{K}$ (see VDOBE). When the absorption profile is dominated by thermal Doppler broadening, the resolution $\Delta y$ which can be achieved on the disk is given by

$$
\frac{\Delta y}{2 R_{*}}=1.28 \frac{\sqrt{T_{4}}}{\left|v_{*, 10} \sin i\right|} f\left(\tau_{0}\right)
$$

where $T_{4}$ is the cloud temperature in units of $10^{4} \mathrm{~K}$ and $v_{*, 10}$ is the intrinsic equatorial rotation speed of the star in units of $10 \mathrm{~km} / \mathrm{s} ; f\left(\tau_{0}\right)$ is a slowly varying function of the central optical depth $\tau_{0}$ (for $\tau_{0} \ll 1 f=0.83$ and for $\tau_{0}=10$ $f=1.63)$. For clouds to show in- and egress requires that $\Delta y / 2 R_{*}<1$. This condition is only fulfilled for rapidly rotating stars, like HK Aqr and AB Dor, and for stars for which the inclination is not too low. It could well be that the cloud phenomenon is not restricted to fast rotators, but that only for fast rotators can it be identified because of the presence of discrete absorptions. In other stars it will show up as variations of $\mathrm{H}_{\alpha}$ affecting the whole line profile. 


\section{The Nature of the Clouds}

In the previous section we showed that, based on spectral diagnostics, clouds do not necessarily have to be an exclusive phenomenon for rapid rotators. Clouds were first detected on the rapidly rotating star AB Dor (Cameron and Robinson $1989 \mathrm{a}, \mathrm{b}$ ). Based on the technique of skew-mapping these authors found evidence of clouds beyond the co-rotation radius. In that case fast rotation could be important for cloud formation and Cameron and Robinson suggested that the clouds form in loops with temperature inversions. These can be created when the effective gravity changes direction in a loop, e.g., at the co-rotation radius. We note that the $\mathrm{AB}$ Dor clouds found with the more traditional technique of gaussian fitting are mainly below the co-rotation radius. The clouds near HK Aqr are also mainly found below the co-rotation radius but still at some considerable distance of the star. Because these clouds cannot be formed by gravity reversals, it is likely that they arise because of insufficient coronal heating near the apex of loops, leading to a temperature inversion. To suppress the Rayleigh-Taylor instability a dip is required in the magnetic field making the clouds resemble filament-like structures. We can consider two possible topologies. In the normal polarity model (NP) the Lorentz force of the background magnetic field balances the effective gravity while in the inverse polarity model (IP) the Lorentz force of the background field is balanced by the Lorentz force due to a surface current distribution on the star. If we assume that the clouds form between the star and the co-rotation radius, and then move slowly outward, then the NP model has the undesired property that the filament current has to decrease as the filament moves outward and has to change sign as the cloud passes through the co-rotation radius. For the IP model this problem does not exist because the current increases as the cloud moves outward. Also, NP prominences are unstable to radial perturbations while IP prominences are not. If the clouds are related to IP prominences then there is no reason why their presence should be restricted to rapid rotators, since for IP prominences mechanical forces are unimportant for the equilibrium. For weakly ionized clouds the typical life time of a cloud is $\tau \approx 21.6\left(\ell_{c} / R_{*}\right)^{2} n_{n, 10}$ hours and the velocity of the cloud with respect to the field is $v_{\perp} \approx 5\left(\ell_{c} / R_{*}\right)^{-1} n_{n, 10}^{-1} \mathrm{~km} / \mathrm{s}$. Here $\ell_{c}$ is the typical dimension of the cloud and $n_{n, 10}$ is the density of the neutrals in units of $10^{10} \mathrm{~cm}^{-3}$.

\section{References}

Byrne, P.B., Eibe, M.T. and Rolleston, W.R.J. 1996, A\&A, 311, 651

Cameron, A.C. and Robinson, R. 1989a, MNRAS, 236, 57

Cameron, A.C. and Robinson, R. 1989b, MNRAS, 238, 657

van den Oord, G.H.J., Byrne, P.B. and Eibe, M.T 1997, A\&A, submitted 